\title{
STOCHASTIC MODELS FOR A CHEMOSTAT AND LONG-TIME BEHAVIOR
}

\author{
PIERRE COLLET, ${ }^{*}$ École Polytechnique \\ SERVET MARTÍNEZ, ${ }^{* * * * *}$ Universidad de Chile \\ SYLVIE MÉLÉARD, ${ }^{* * * *}$ École Polytechnique \\ JAIME SAN MARTÍN, ${ }^{* * * * * * *}$ Universidad de Chile
}

\begin{abstract}
We introduce two stochastic chemostat models consisting of a coupled population-nutrient process reflecting the interaction between the nutrient and the bacteria in the chemostat with finite volume. The nutrient concentration evolves continuously but depends on the population size, while the population size is a birth-and-death process with coefficients depending on time through the nutrient concentration. The nutrient is shared by the bacteria and creates a regulation of the bacterial population size. The latter and the fluctuations due to the random births and deaths of individuals make the population go almost surely to extinction. Therefore, we are interested in the long-time behavior of the bacterial population conditioned to nonextinction. We prove the global existence of the process and its almost-sure extinction. The existence of quasistationary distributions is obtained based on a general fixed-point argument. Moreover, we prove the absolute continuity of the nutrient distribution when conditioned to a fixed number of individuals and the smoothness of the corresponding densities.
\end{abstract}

Keywords: Population process; chemostat; extinction; quasistationary distribution

2010 Mathematics Subject Classification: Primary 60J25; 60J27; 92D25

\section{Introduction}

For some decades, since the first work of Monod [9] and Novik and Szilar [10], [11], see also [12], biologists have developed procedures which allow them to maintain a bacterial population at a stationary finite size while at the same time keep a constant individual growth rate of bacteria. The procedure is based on a chemostat: bacteria live in a growth container of constant volume in which liquid is injected continuously. The entering liquid contains a fixed concentration of nutrient but no bacteria (fresh liquid). In the container, the nutrient is consumed by the bacteria. We assume that the chemostat is well stirred, so that the distribution of bacteria and nutrient are spatially uniform. Since the container has a finite volume and fresh liquid continuously enters, an equal amount of liquid pours out, containing both unconsumed nutrients and bacteria.

\footnotetext{
Received 21 June 2012; revision received 11 October 2012.

* Postal address: Centre de Physique Théorique, CNRS UMR 7644, École Polytechnique, F-91128 Palaiseau Cedex, France. Email address: collet@cpht.polytechnique.fr

** Postal address: Departamento Ingeniería Matemática and Centro Modelamiento Matemático, Universidad de Chile, UMI 2807 CNRS, Casilla 170-3, Correo 3, Santiago, Chile.

*** Email address: smartine@dim.uchile.cl

**** Postal address: CMAP, École Polytechnique, CNRS, route de Saclay, 91128 Palaiseau Cedex, France.

Email address: sylvie.meleard@polytechnique.edu

***** Email address: jsanmart@dim.uchile.cl
} 
These chemostats are extremely useful in the study of bacterial population dynamics, in particular in the study of the selection of the fastest growing species or the fixation of advantageous mutations (see [4], [5], [6], [7], or [8]). In the literature, their study is mainly based on deterministic models where both nutrient and bacteria population dynamics are described by a coupled deterministic continuous process. Deterministic approximations for the bacterial population's size are justified by a large population assumption.

In this work we develop stochastic chemostat models based on a previous work of Crump and O'Young [3], taking into account the fact that the bacteria population may not be large enough to justify a deterministic approximation. We introduce a coupled population-nutrient process reflecting the interaction between the nutrient and the bacteria in the chemostat. The nutrient concentration evolves continuously but depends on the population size, while the population size is a birth-and-death process with coefficients depending on time through the nutrient concentration. Moreover, the time derivative of the nutrient concentration jumps simultaneously with the population size.

We thus take into account the random fluctuations of this population size due to the individual births and deaths. The bacteria need nutrient to reproduce. We will consider two cases. In the first case the bacterial population dies instantaneously if the nutrient is missing. In the second case bacteria can survive without nutrient by undergoing some kind of hibernation and may wake up once nutrient reappears. The nutrient is shared by the bacteria. This creates an indirect competition between bacteria and leads to a regulation of their population size. In our models, the fluctuations due to the random births and deaths of individuals and the size regulation make the population go almost surely to extinction. Therefore, the long-time behavior of the population's size is obvious and the interesting questions concern firstly the rate of extinction and secondly the long-time behavior conditioned to nonextinction which is captured by the notion of a quasistationary distribution.

To our knowledge, the models introduced in this paper are the first stochastic chemostat models where interaction between bacteria is taken into account, leading to extinction. The study of quasistationarity nevertheless gives a description of a quasistability which can happen on a faster time scale than extinction. This work concerns monotype individuals, but could be generalized to a multitype population.

In Section 2 we describe the two population-nutrient models described above and prove in Section 3 their global existence. We also show the extinction of the population when time increases. The existence of quasistationary distributions is obtained in Section 4. Our main theorem is based on a general argument proved in [2]. In Section 5 we prove the absolute continuity of the nutrient distribution when conditioned to a fixed number of individuals and the smoothness of the corresponding densities.

\section{The stochastic population-nutrient process}

We consider a stochastic discrete population process describing the dynamics of a bacteria population for which individuals develop and reproduce depending on the quantity of nutrient $y$ in the solution. The dynamics of the nutrient are related to the consumption of the individuals. We assume that the concentration of nutrient in the injected solution (without bacteria) is a constant equal to $y^{*}$. The chemostat has a finite volume equal to 1 . The liquid enters in the chemostat free of bacteria and pours out after being well stirred in the container. The liquid poured out contains bacteria. The dilution coefficient of nutrient in the fresh liquid per unit of time is $D$. Since the liquid is well stirred, around $N(t) D$ bacteria will be washed out in the 
pouring out, where $N(t)$ is the size of the bacteria population at time $t$. Thus, $D$ is also the rate at which an individual will disappear due to the evacuation of liquid.

We consider coupled processes in which the nutrient concentration evolves continuously while the bacteria population size evolves as a time-continuous birth-and-death process with coefficients depending on the nutrient concentration. We assume that the nutrient is partly consumed during the reproduction of bacteria.

We will denote by $(Y(t), t \geq 0)$ the concentration of nutrient and by $(N(t): t \geq 0)$ the population size process. The stochastic process $Z=(Z(t):=(N(t), Y(t)): t \geq 0)$ describes both the population size and the nutrient concentration in the chemostat.

Let us now define the parameters of the model.

If $y$ is the quantity of nutrient then the birth and death parameters driving the dynamics of the population are as follows.

- The birth rate per individual is $b(y)$, where the function $b: \mathbb{R}_{+} \rightarrow \mathbb{R}_{+}$is assumed to be an increasing continuous function and such that $b(0)=0$ and $b(y)>0$ for $y>0$. We assume that $b$ is bounded with an upper bound $b_{\infty}$.

A usual example of a function $b$ is, for some constant $K>0$,

$$
b(y)=b_{\infty} \frac{y}{K+y} .
$$

An extra hypothesis that we will add for some results is that $b$ is differentiable and $\mathrm{d} b(0) / \mathrm{d} y>0$.

- The background death rate per individual is $d(y)$, so it is supposed to be a function of the concentration of nutrient. The function $d: \mathbb{R}_{+} \rightarrow \mathbb{R}_{+} \cup\{\infty\}$ is assumed to be continuous, nonincreasing, and strictly positive, and $d(0)$ is the unique value that can be infinite.

- The dilution makes each individual disappear at rate $D$ independently of the birth and death events.

- The per individual rate of consumption of nutrient for reproduction is $b(y) / R$, where $R$ denotes the biomass yield. Furthermore, individuals consume nutrient during their life and the quantity of nutrient consumed per individual will be denoted by $\eta \geq 0$.

We will consider two cases. In the first case, individuals need nutrient to survive. Then we will assume that their death is instantaneous as soon as nutrient is missing; therefore, $d(0)=\infty$.

In the second case, bacteria enter in some kind of hibernation if nutrient is missing. That means that $d(0)$ can be finite. In both cases, we will set

$$
\tilde{b}(y)=\frac{b(y)}{R}+\eta \mathbf{1}_{\{y>0\}} .
$$

Let us now describe the process. In both cases the nutrient concentration process $Y=$ $(Y(t): t \geq 0)$ evolves according to

$$
\frac{\mathrm{d} Y(t)}{\mathrm{d} t}=D\left(y^{*}-Y(t)\right)-\tilde{b}(Y(t)) N(t) .
$$

The process $Z$ has the following infinitesimal generator: for $(n, y) \in \mathbb{N} \times \mathbb{R}_{+}$,

$$
\begin{aligned}
\mathcal{L} f(n, y)= & b(y) n f(n+1, y)+(D+d(y)) n f(n-1, y) \\
& -(b(y)+D+d(y)) n f(n, y) \\
& +\left(D\left(y^{*}-y\right)-n \tilde{b}(y)\right) \partial_{y} f(n, y) .
\end{aligned}
$$

We refer the reader to [1] for the numerical study of similar models. 
Below, we show that the hypotheses we gave on the coefficients leading the process guarantee that $Z$ is well defined and takes values in $\mathbb{N} \times\left[0, y^{*}\right]$.

Proposition 2.1. The process $Z$ is well defined and takes values in $\mathbb{N} \times \mathbb{R}_{+}$for all positive time $t \in \mathbb{R}_{+}$. Moreover, $\mathbb{N} \times\left[0, y^{*}\right]$ is an invariant set for the process $Z$, so $Y(0) \in\left[0, y^{*}\right]$ implies that $Y(t) \in\left[0, y^{*}\right]$ for all $t \in \mathbb{R}_{+}$.

Proof. The process $N=(N(t): t \geq 0)$ has a pathwise representation driven by a point Poisson measure $\mathcal{N}(\mathrm{d} \theta, \mathrm{d} s)$ defined on $\mathbb{R}_{+} \times \mathbb{R}_{+}$:

$$
\begin{aligned}
& N(t)=N_{0}+\int_{\mathbb{R}_{+} \times(0, t]} \mathbf{1}_{\left\{\theta \leq b\left(Y_{s}\right)\right\}} \mathcal{N}(\mathrm{d} \theta, \mathrm{d} s)-\int_{\mathbb{R}_{+} \times(0, t]} \mathbf{1}_{\left\{b\left(Y_{s}\right) \leq \theta \leq b\left(Y_{s}\right)+D+d\left(Y_{s}\right)\right\}} \mathcal{N}(\mathrm{d} \theta, \mathrm{d} s), \\
& Y(t)=Y(0)+\int_{0}^{t}\left(D\left(y^{*}-Y(s)\right)-\tilde{b}(Y(s)) N_{s}\right) \mathrm{d} s .
\end{aligned}
$$

It is obvious that the process $N$ is stochastically upper bounded by a birth process with individual birth rate $b_{\infty}$. Since the birth process with constant rate has no explosion, the process $N$ does not explode.

Let us now study the nutrient concentration $Y=(Y(t): t \geq 0)$. Note that the assumptions on the parameters and (2.1) ensure that $Y(t) \geq 0$. Indeed, the derivative of $Y(t)$ at $y=0$ cannot be negative.

Let us show that $\mathbb{N} \times\left[0, y^{*}\right]$ is invariant. Take $Y(0) \in\left[0, y^{*}\right]$. A standard comparison theorem yields $Y(t) \leq v(t)$, where $v^{\prime}(t)=D\left(y^{*}-v(t)\right), v(0)=Y(0)$. But, in that case, $v(t)=y^{*}-\left(y^{*}-Y(0)\right) \mathrm{e}^{-D t} \leq y^{*}$, so it remains in $\left[0, y^{*}\right]$ forever, proving the invariance.

For the initial condition $N(0) \in \mathbb{N}^{*}$ and $Y(0) \in \mathbb{R}_{+} \backslash\left[0, y^{*}\right]$, at time $t=\left(Y(0)-y^{*}\right) / \tilde{b}\left(y^{*}\right)$ the process has already attained the invariant set $\left[0, y^{*}\right] \times \mathbb{R}_{+}$or become extinct.

\section{Study of extinction}

We are now interested in studying the extinction of the population or the complete consumption of the nutrient or other specific states of the population-nutrient process and the associated hitting times.

Let $\mathcal{B}\left(\mathbb{N} \times \mathbb{R}_{+}\right)$be the class of Borel sets of $\mathbb{N} \times \mathbb{R}_{+}$, and let $\mathcal{B}\left(\mathbb{R}_{+}\right)$be the class of Borel sets of $\mathbb{R}_{+}$. For $D \in \mathscr{B}\left(\mathbb{N} \times \mathbb{R}_{+}\right)$, we denote by $T^{D}=\inf \{t \geq 0: Z(t) \in D\}$ the hitting time of $D$ by the process, with the usual convention that inf $\varnothing=\infty$. Let

$$
T_{0}=T^{\{0\} \times \mathbb{R}_{+}}=\inf \{t \geq 0: N(t)=0\}
$$

be the killing time for the process $N$. We also denote by $T_{\leq m}=T^{\{0, \ldots, m\} \times \mathbb{R}_{+}}=$ $\inf \{t \geq 0: N(t) \leq m\}$, and similarly for $T_{<m}$.

Note that the set $\{0\} \times \mathbb{R}_{+}$is an absorbing set, that is, $N(t)=0$ for all $t \geq T_{0}$.

After $T_{0}$ the nutrient $Y(t)$ is absorbed linearly at $y^{*}$, in fact, $Y(t)=y^{*}$ for $t \geq T_{0}+\left(y^{*}-\right.$ $\left.Y\left(T_{0}\right)\right) / D y^{*}$ and $Y(t)=Y\left(T_{0}\right)+D\left(t-T_{0}\right)$ for $t \in\left[T_{0}, T_{0}+\left(y^{*}-Y\left(T_{0}\right)\right) / D\right]$.

Let us first study the stationary nutrient concentration states at a fixed population size.

Lemma 3.1. Consider the equation

$$
G_{n}(y)=0 \quad \text { with } \quad G_{n}(y):=D\left(y^{*}-y\right)-n \tilde{b}(y) .
$$

(i) If $\eta=0$ then, for any $n \in \mathbb{N}$, (3.1) has a unique simple root $y_{n}$ which belongs to $\left[0, y^{*}\right]$. In addition, the sequence $\left(y_{n}: n \in \mathbb{N}^{*}\right)$ of the roots decreases to 0 .

(ii) If $\eta>0$ then (3.1) has no root for $n>D y^{*} / \eta$ and admits a simple root $y_{n}$ for $n \leq D y^{*} / \eta$. 
Proof. Let us fix $n$. Obviously, for $n=0$, (3.1) has the trivial root $y_{0}=y^{*}$, so we restrict $n$ to be in $\mathbb{N}^{*}$. By assumption, the function $G_{n}$ is strictly decreasing, so, for each $n \in \mathbb{N}^{*}$, there exists at most one root. Note that, for all such $n$, we have $G_{n}\left(y^{*}\right)<0$ and so there is no root to $(3.1)$ in the set $\left[y^{*}, \infty\right)$.

Assume that $\eta=0$. For all $n$, we have $G_{n}(0)>0$. Then there exists a unique root, denoted by $y_{n}$, which satisfies $G_{n}\left(y_{n}\right)=0$. On the other hand, we have

$$
G_{n+1}\left(y_{n}\right)=D\left(y^{*}-y_{n}\right)-(n+1) \tilde{b}\left(y_{n}\right)=-\tilde{b}\left(y_{n}\right)
$$

Then, $G_{n+1}(0)>0$ and $G_{n+1}\left(y_{n}\right)<0$. We deduce that $0<y_{n+1}<y_{n}<y^{*}$. Let $y_{\infty}=\lim _{n \rightarrow \infty} y_{n}$. By continuity,

$$
D\left(y^{*}-y_{\infty}\right)-n \tilde{b}\left(y_{\infty}\right)=\lim _{n \rightarrow \infty} D\left(y^{*}-y_{n}\right)-n \tilde{b}\left(y_{n}\right)=0 .
$$

Then, necessarily, $\tilde{b}\left(y_{\infty}\right)=0$ and so $y_{\infty}=0$ and $b\left(y_{n}\right) \sim D y^{*} R / n$ as $n \rightarrow \infty$.

Assume that $\eta>0$. Then, for all $n>D y^{*} / \eta$, we have $G_{n}(0)<0$ and so (3.1) has no solution. Hence, the same argument as before gives the existence of a finite set of roots ( $\left.y_{n}: 1 \leq n \leq\left\lfloor D y^{*} / \eta\right\rfloor\right)$ decreasing with $n$, where $\left\lfloor D y^{*} / \eta\right\rfloor$ is the largest integer that is smaller or equal to $D y^{*} / \eta$.

Note that, when $n_{0}=D y^{*} / \eta$, then $y_{n_{0}}=0$ and, if $D y^{*}<\eta$, there is no root.

From Lemma 3.1, we know that the set

$$
\S=\left\{y \in \mathbb{R}_{+}: \text {there exists } n \in \mathbb{N}^{*}, D\left(y^{*}-y\right)-\tilde{b}(y) n=0\right\}
$$

is a countable set included in $\left[0, y^{*}\right)$. If $\eta=0$, it is infinite and accumulates at 0 and, if $\eta>0$, it is finite.

In the sequel, when we refer to $y_{n}$, we will implicitly assume that it exists, namely we are in the case $\eta=0$ or $\eta>0$, but $n \in\left\{1, \ldots,\left\lfloor D y^{*} / \eta\right\rfloor\right\}$.

Corollary 3.1. (i) The set $\mathbb{N} \times\left[0, y_{1}\right]$ is invariant for the process $Z^{T_{0}}=\left(Z(t): t \leq T_{0}\right)$ up to extinction, that is, if $Z(0)=(N(0), Y(0)) \in \mathbb{N} \times\left[0, y_{1}\right]$ then $Z(t)=(N(t), Y(t)) \in \mathbb{N} \times\left[0, y_{1}\right]$ for all $t \leq T_{0}$.

(ii) The set $\mathbb{N} \times\left[0, y_{n}\right]$ is invariant for the process $Z^{T_{<n}}=\left(Z(t): t \leq T_{<n}\right)$.

Proof. Let us show the first part. We have $Z(t)=(N(t), Y(t)) \in \mathbb{N}^{*} \times\left[0, y_{1}\right]$ for all $t<T_{0}$ because $\mathrm{d} Y(t) / \mathrm{d} t \leq 0$ when $y \geq y_{1}$ and $n \geq 1$, and so if the trajectory arrives to $y_{1}$, the variable $Y(t)$ immediately decreases. For $n=0$, it is evident, because we stop the process at $T_{0}$, and so $Y\left(T_{0}\right)=Y\left(T_{0}^{-}\right)$but $Y\left(T_{0}^{-}\right) \leq y_{1}$ since $N\left(T_{0}^{-}\right)=1$.

The proof of part (ii) is shown in a similar way.

Let us state a useful lemma.

Lemma 3.2. For any $n_{0} \in \mathbb{N}^{*}$, there exists $t_{0}>0$ such that

$$
\inf _{y \in\left[0, y^{*}\right], 1 \leq n \leq n_{0}} \mathbb{P}_{(n, y)}\left(T_{0}<t_{0}\right)>0 .
$$

Proof. The proof follows at once from the fact that the population process is stochastically dominated by a birth-and-death process with birth rate $b\left(y^{*}\right)$ and death rate $D+d\left(y^{*}\right)$. 


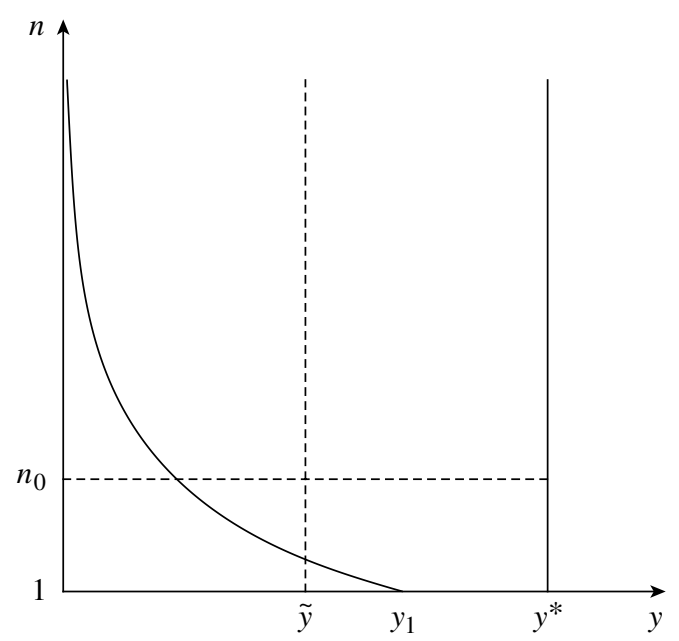

FIGURE 1: The curve $y(t)$.

Theorem 3.1. We have extinction of the population almost surely, namely, for any $y \in\left[0, y^{*}\right]$ and any integer $n$,

$$
\mathbb{P}_{(n, y)}\left(T_{0}<\infty\right)=1 \text {. }
$$

Proof. Note that an obvious comparison theorem as used in the previous proof cannot be applied. Indeed, the birth and death rates $b\left(y^{*}\right)$ and $D+d\left(y^{*}\right)$ could correspond to a supercritical case. The effect of the chemostat through the nutrient is a regulation of the population.

We will exhibit an integer $n_{0}$ such that the population process will spend an infinite amount of time below $n_{0}+1$.

Let $\tilde{y}>0$ be such that $b(\tilde{y})<D+d(\tilde{y})$. Note that, by monotonicity, for all $y \in[0, \tilde{y}]$, we have $b(y)<D+d(y)$. Let us define $n_{0}$ as an integer such that $y_{n_{0}}<\tilde{y}$ if some exist or equal to $\left[D y^{*} / \tilde{b}(0)\right]+1$ otherwise (see Figure 1 ). Let $\tau$ be the random time defined by

$$
\sup _{t \geq \tau} N(t) \leq n_{0}
$$

Then, it follows from Lemma 3.2 and the Markov property that if $\mathbb{P}_{(n, y)}(\tau<\infty)>0$ then

$$
\mathbb{P}_{(n, y)}\left(T_{0}<\infty \mid \tau<\infty\right)=1 .
$$

Assume now that $\mathbb{P}_{(n, y)}(\tau=\infty)=1$. Let $\tau^{\prime}$ be the random time defined by

$$
\inf _{t \geq \tau^{\prime}} N(t)>n_{0}
$$

Assume that $\mathbb{P}_{(n, y)}\left(\tau^{\prime}<\infty\right)>0$. Let $y_{0}(t)$ be the solution of the differential equation

$$
\frac{\mathrm{d} y_{0}}{\mathrm{~d} t}=D\left(y^{*}-y_{0}(t)\right)-\left(n_{0}+1\right) \tilde{b}\left(y_{0}(t)\right)
$$

with initial condition $y_{0}(0)=y^{*}$. Let $t_{1}>0$ be the finite solution of

$$
y_{0}\left(t_{1}\right)=\tilde{y} .
$$


The time $t_{1}$ is finite from the choice of $n_{0}$. It is easy to verify that, for any integer-valued measurable function $n(t) \geq n_{0}+1$, the solution $y(t)$ of the equation

$$
\frac{\mathrm{d} y}{\mathrm{~d} t}=D\left(y^{*}-y(t)\right)-n(t) \tilde{b}(y(t))
$$

with initial condition $y(0) \leq y^{*}$, satisfies

$$
y(t) \leq \tilde{y}
$$

for any $t \geq t_{1}$. On the set $\left\{\tau^{\prime}<\infty\right\}$, the process $\left(N(t), t \geq \tau^{\prime}+t_{1}\right)$ is dominated by a linear birth-and-death process with birth rate $b(\tilde{y})$ and death rate $D+d(\tilde{y})$ (from the monotonicity of the functions). This birth-and-death chain attains $n_{0}$ almost surely in finite time since $b(\tilde{y})<D+d(\tilde{y})$; see [13]. Hence, on $\left\{\tau^{\prime}<\infty\right\}$, the process $\left(N(t), t \geq \tau^{\prime}+t_{1}\right)$ should also attain $n_{0}$ in finite time. This contradicts our assumption that $\mathbb{P}_{(n, y)}\left(\tau^{\prime}<\infty\right)>0$.

It remains to consider the case $\tau=\tau^{\prime}=\infty$ almost surely. In this case there exist two infinite sequences of random times

$$
T_{1}<S_{1}<T_{2}<S_{2}<\cdots
$$

such that

$$
N(t) \leq n_{0} \quad \text { for } t \in\left[T_{j}, S_{j}\right), \quad N(t)>n_{0} \quad \text { for } t \in\left[S_{j}, T_{j+1}\right) .
$$

Since we visit the set $\left\{N \leq n_{0}\right\}$ infinitely many times, and at each visit we have a uniformly positive probability of extinction, it follows by the Markov property and the Borel Cantelli lemma that

$$
\mathbb{P}_{(n, y)}\left(T_{0}<\infty \mid \tau=\tau^{\prime}=\infty\right)=1 .
$$

One of our main objectives of this work is to study the processes up to the moment the population is extinct, $Z^{T_{0}}=\left(Z(t): t \leq T_{0}\right)$, or before the moment of extinction, $Z^{T_{0}^{-}}=$ $\left(Z(t): t<T_{0}\right)$. All the statements related to quasistationary distributions depend on $Z^{T_{0}^{-}}$.

\section{Existence of quasistationary distributions}

A quasistationary distribution $v$ (with respect to the absorbing time $T_{0}$ ) is a probability measure defined on $\mathbb{N}^{*} \times \mathbb{R}_{+}$that verifies

$$
\mathbb{P}_{v}\left(Z(t) \in D \mid T_{0}>t\right)=v(D) \quad \text { for all } t \geq 0 \text { and all } D \in \mathscr{B}\left(\mathbb{N}^{*} \times \mathbb{R}_{+}\right) .
$$

It is known that starting from a quasistationary distribution, the time of absorption is exponential, that is, $\mathbb{P}_{v}\left(T_{0}>t\right)=\mathrm{e}^{-\lambda t}$, where $\lambda=\lambda(\nu)>0$. Indeed, the strong Markov property implies that

$$
\begin{aligned}
\mathbb{P}_{v}\left(T_{0}>t+s\right) & =\mathbb{P}_{v}\left(T_{0}>t, \mathbb{P}\left(T_{0}>t+s \mid \mathcal{F}_{t}\right)\right) \\
& =\mathbb{P}_{v}\left(T_{0}>t, \mathbb{P}_{Y_{t}}\left(T_{0}>s\right)\right) \\
& =\mathbb{P}_{v}\left(T_{0}>s\right) \mathbb{P}_{v}\left(T_{0}>t\right),
\end{aligned}
$$

where $\left(\mathcal{F}_{t}: t \geq 0\right)$ is the natural filtration for $Z$. From this equality, the distribution of $T_{0}$ under $\mathbb{P}_{v}$ is exponential with parameter $\lambda(v)$. The fact that $\lambda(v)>0$ follows from the fact that absorption is certain. 
Let $\mathcal{M}_{b}=\left\{f: \mathbb{N}^{*} \times \mathbb{R}_{+} \rightarrow \mathbb{R}\right.$ bounded and measurable $\}$. Equation (4.1) can be written as, there exists $\lambda>0$ such that

$$
\mathbb{E}_{v}\left(f(Z(t)), T_{0}>t\right)=\mathrm{e}^{-\lambda t} \int_{0}^{\infty} f(y) v(\mathrm{~d} y) \text { for all } t>0, f \in \mathcal{M}_{b} .
$$

Define

$$
\kappa_{n}=v\left(\{n\} \times \mathbb{R}_{+}\right)=\mathbb{P}_{v}(N(0)=n),
$$

and define by

$$
v_{n}(B)=v\left(\{n\} \times B \mid\{n\} \times \mathbb{R}_{+}\right)=\mathbb{P}_{v}(Y(0) \in B \mid N(0)=n) \quad \text { for all } B \in B_{\left(\mathbb{R}_{+}\right)}
$$

the probability measure conditioned to have $n$ individuals. Then

$$
v(D)=\sum_{n \in \mathbb{N}^{*}} \kappa_{n} v_{n}\left(D \cap\{n\} \times \mathbb{R}_{+}\right), \quad v_{n}\left(\mathbb{R}_{+}\right)=1 \text { for all } n \in \mathbb{N}^{*} \text { and } \sum_{n \in \mathbb{N}^{*}} \kappa_{n}=1 .
$$

In order that the probability measure $v$ is a quasistationary distribution, it must satisfy the infinitesimal condition deduced from (4.2), which is given by, there exists $\lambda>0$ such that

$$
\sum_{n \in \mathbb{N}^{*}} \kappa_{n} \int_{0}^{\infty} \mathrm{d} v_{n}(y)[\mathcal{L} f(n, y)-\lambda f(n, y)]=0 \quad \text { for all } f \in \mathcal{M}_{b} .
$$

For the adjoint operator $\mathcal{L}^{\dagger}$ of $\mathcal{L}$ defined in (2.2) to be a quasistationary distribution we have, there exists $\lambda>0$ such that

$$
\mathcal{L}^{\dagger} v=-\lambda v
$$

Theorem 4.1. Assume that there exists $0 \leq \sigma<1$ such that $\lim \sup _{y \searrow 0} y^{\sigma} d(y)<\infty$. Then there exists a quasistationary distribution. Moreover, there exists at least a quasistationary distribution such that $Y$ is supported in $\left[0, y^{*}\right]$.

Proof. It suffices to show the existence of a quasistationary distribution. In fact, the last part of the statement follows when the existence proof is applied to the process $Z$ taking values in the invariant set $\mathbb{N} \times\left[0, y^{*}\right]$.

The idea for showing the existence is to use the abstract Theorem 4.2 proved in [2]. We assume that $y \in\left[0, y^{*}\right]$. We define a function

$$
\varphi_{1}(y, n)=\mathbf{1}_{\{n \geq 1\}} .
$$

Then a simple computation (the same as for a birth-and-death process since $\varphi_{1}$ does not depend on $y$ ) leads to

$$
\mathcal{L} \varphi_{1}(y, n)= \begin{cases}0 & \text { if } n>1 \\ -D-d(y) & \text { if } n=1\end{cases}
$$

Therefore, if $d$ is bounded above by a constant $d^{*}$ then

$$
\mathrm{e}^{-t\left(D+d^{*}\right)} \varphi_{1} \leq \mathrm{e}^{t \mathcal{L}} \varphi_{1} \leq \varphi_{1}
$$

If $d$ is not bounded, we will prove the lower bound

$$
\inf _{n_{0} \geq 1, y_{0} \in\left[0, y^{*}\right]} \mathbb{P}_{n_{0}, y_{0}}(N(1) \geq 1) \geq Q \text { for a constant } Q>0 .
$$


By the Markov property, it suffices to prove that there exists $Q>0$ such that

$$
\inf _{y_{0} \in\left[0, y^{*}\right]} \mathbb{P}_{1, y_{0}}(N(t)=1 \text { for all } 0 \leq t \leq 1) \geq Q .
$$

Since $N(t)=1$ on the whole time interval $[0,1], y(t)$ satisfies the differential equation

$$
\frac{\mathrm{d} y}{\mathrm{~d} t}=D\left(y^{*}-y\right)-\tilde{b}(y)
$$

with initial condition $y_{0}$. Since there is no birth and no death in the time interval $[0,1]$, we obtain

$$
\mathbb{P}_{1, y_{0}}(N(t)=1 \text { for all } 0 \leq t \leq 1)=\exp \left(-\int_{0}^{1}(d(y(t))+b(y(t))) \mathrm{d} t\right) .
$$

Since $b(y)$ is bounded uniformly in $y$, the above quantity does not vanish if

$$
\int_{0}^{1} d(y(t)) \mathrm{d} t<\infty
$$

It is easy to show that there exists a constant $c>0$ such that, for any $y^{*} \geq y_{0} \geq 0$, we have $y(t) \geq c t$ for any $t \in[0,1]$. Since $d(y) \leq \mathcal{O}(1) y^{-\sigma}$ for small $y$, we obtain

$$
\int_{0}^{1} d(y(t)) \mathrm{d} t \leq \mathcal{O}(1) \int_{0}^{1} y(t)^{-\sigma} \mathrm{d} t<\infty .
$$

It now immediately follows that

$$
Q \varphi_{1} \leq \mathrm{e}^{\mathcal{L}} \varphi_{1} \leq \varphi_{1}
$$

The second function is

$$
\varphi_{2}(y, n)=\mathbf{1}_{\{n \geq 1\}} \mathrm{e}^{a(y) n},
$$

with $a(y)=\alpha y+a_{0}, \alpha>0$, and $a_{0}>0$. A simple computation for $n>1$ (same as for a birth-and-death process since $\varphi_{2}$ does not depend on $y$ ) leads to

$$
\mathcal{L} \varphi_{2}(y, n)=n \mathrm{e}^{a(y) n} \Xi(n, y),
$$

where

$$
\Xi(n, y)=b(y)\left(\mathrm{e}^{a(y)}-1-\frac{\alpha n}{R}\right)+(D+d(y))\left(\mathrm{e}^{-a(y)}-1\right)+D \alpha\left(y^{*}-y\right)-\alpha \eta \mathbf{1}_{\{y>0\}} .
$$

Let us show that there exist $A>0$ and $N_{0}$ such that, for all $n>N_{0}$,

$$
\sup _{y \in\left[0, y^{*}\right]} \Xi(n, y) \leq-A \text {. }
$$

Define $\zeta(y)=(D+d(y))\left(\mathrm{e}^{-a(y)}-1\right)+D \alpha\left(y^{*}-y\right)-\alpha \eta \mathbf{1}_{\{y>0\}}$. We choose $a_{0}$ and $\alpha$ such that $D\left(\mathrm{e}^{-a_{0}}-1\right)+D \alpha y^{*}<0$. Then $\zeta(0)<0$. It follows that, for all $y \in\left[0, y^{*}\right], \zeta(y)<-A$ for some $A>0$.

Consider $\hat{N}_{0}$ such that $\mathrm{e}^{a\left(y^{*}\right)}-1-\alpha \hat{N}_{0} / R<0$. We still have, for $n>\hat{N}_{0}, \mathrm{e}^{a\left(y^{*}\right)}-1-$ $\alpha n / R<0$. Then, for $n>\hat{N}_{0}$, we obtain $\Xi(n, y) \leq-A$. Therefore, for any $C>0$, there exists $N(C)$ such that, for any $n>N(C)$,

$$
\mathcal{L} \varphi_{2}(y, n) \leq-C \varphi_{2}(y, n) .
$$

Therefore, for any $C>0$, there exists $\Gamma(C)>0$ (finite) such that

$$
\mathcal{L} \varphi_{2} \leq-C \varphi_{2}+\Gamma(C) \varphi_{1}
$$


(the estimate for $n=1$ is by direct computation, taking $\Gamma(C)$ adequately large enough). Hence,

$$
\mathrm{e}^{t \mathcal{L}} \varphi_{2} \leq \mathrm{e}^{-t C} \varphi_{2}+\frac{\Gamma(C)}{C} \varphi_{1} .
$$

In the case where $d$ is bounded above by $d^{*}<\infty$, we now choose $C>D+d^{*}$ and $t=1$ and apply Theorem 4.2 of [2]. In the case where $d$ is unbounded, we choose $C>-\log Q$ and apply Theorem 4.2 of [2].

\section{Properties of the quasistationary distributions}

Proposition 5.1. Any quasilimiting distribution has support in $\mathbb{N}^{*} \times\left[0, y^{*}\right]$.

Proof. We recall that a quasilimiting distribution $v$ is a probability measure on $\mathbb{N}^{*} \times \mathbb{R}_{+}$ such that, for some initial point $\left(n_{0}, y_{0}\right) \in \mathbb{N}^{*} \times \mathbb{R}_{+}$,

$$
v(A)=\lim _{t \rightarrow \infty} \mathbb{P}_{\left(n_{0}, y_{0}\right)}\left(Z(t) \in A \mid T_{0}>t\right) \quad \text { for all } A \in \mathcal{B}\left(\mathbb{N}^{*} \times \mathbb{R}_{+}\right) .
$$

If $y_{0} \in\left[0, y^{*}\right]$, the assertion follows from Proposition 2.1. Let us now assume that $y_{0}>y^{*}$. We introduce the function $t \rightarrow v(t)$ defined by

$$
\frac{\mathrm{d} v}{\mathrm{~d} t}(t)=D\left(y^{*}-v\right)-\tilde{b}\left(y^{*}\right) ; v(0)=y_{0} .
$$

Let $\tau_{0}\left(y_{0}\right)$ be defined by $v\left(\tau_{0}\left(y_{0}\right)\right)=y^{*}$. For $t \leq T_{0} \wedge \tau_{0}\left(y_{0}\right)$, we have $Y(t) \leq v(t)$. It follows from Proposition 2.1 that $\mathbb{P}_{\left(n_{0}, y_{0}\right)}\left(Y(t)>y^{*} \mid T_{0}>t\right)=0$ for all $t \geq \tau_{0}\left(y_{0}\right)$, which concludes the proof.

Theorem 5.1. For all $n \in \mathbb{N}^{*}$ and any quasistationary distribution, the probability measure $v_{n}$ is absolutely continuous with respect to the Lebesgue measure, with $C^{\infty}$-density on the set $\mathbb{R}_{+} \backslash\left\{0, y_{n}\right\}$.

The proof of this theorem is obtained from the following lemmas. Recall that the set 8 has been defined in (3.2).

Lemma 5.1. For all $n \in \mathbb{N}^{*}$, the measure $v_{n}$ satisfies

$$
\mathrm{d} v_{n}=c_{0}^{n} \delta_{0}+\left(\sum_{j \in \mathbb{N}^{*}} c_{j}^{n} \delta_{y_{j}}\right)+u_{n}(y) \mathrm{d} y,
$$

where $c_{j}^{n} \geq 0$ for $j \in \mathbb{N}$, and $u_{n}$ is the density of the absolutely continuous part of $v_{n}$ (so it is a nonnegative integrable function) and it is a $C^{\infty}$-function outside $\& \cup\{0\}$.

Proof. The measures $v_{n}$ satisfy in the sense of distributions the differential equations

$$
\begin{aligned}
& \partial_{y}\left(\left(D\left(y^{*}-y\right)-n \tilde{b}(y)\right) v_{n}\right) \\
& \quad=b(y) n v_{n-1}+(D+d(y)) n v_{n+1}-(b(y)+D+d(y)) n v_{n}+\lambda v_{n} .
\end{aligned}
$$

Since the right-hand side is a measure, we conclude by a recursive argument that the measures $v_{n}$ have a $C^{\infty}$-density on $(\delta \cup\{0\})^{\mathrm{c}}$. This also shows that these measures have no singular part, and the Lebesgue decomposition theorem gives relation (5.1).

Lemma 5.2. Let I be an open interval included in $(\delta \cup\{0\})^{\mathrm{c}}$. If there exists $n \in \mathbb{N}^{*}$ such that $v_{n}(I)=0$ then $v_{j}(I)=0$ for all $j \in \mathbb{N}^{*}$. 
Proof. Assume that there is an interval $I$ not intersecting $\& \cup\{0\}$ such that, for some integer $n \in \mathbb{N}^{*}, v_{n}$ vanishes on $I$. From (5.2) we deduce that $v_{n+1}$ and $v_{n-1}$ also vanish at $I$. Therefore, we conclude recursively that $v_{j}(I)=0$ for all $j \in \mathbb{N}^{*}$.

Lemma 5.3. The probability measure $v_{n}$ is absolutely continuous on $\mathbb{R}_{+} \backslash\left\{0, y_{n}\right\}$ and its density is bounded on any compact set contained in $\mathbb{R}_{+} \backslash\left\{0, y_{n}\right\}$.

Proof. Let us show that if $j \neq n, v_{n}$ cannot have a Dirac mass in $y_{j}$. We proceed by contradiction, so assume that it does. Let $f$ be a $C^{\infty}$-function with compact support containing $y_{j}$ and such that its support does not contain any other point of $S \cup\{0\}$ except $y_{j}$. By using (5.1) we obtain

$$
\begin{aligned}
& -f^{\prime}\left(y_{j}\right)\left(D\left(y^{*}-y_{j}\right)-n \tilde{b}(y)\right)-\int f^{\prime}(y)\left(D\left(y^{*}-y\right)-n \tilde{b}(y)\right) \psi_{n}(y) \mathrm{d} y \\
& =\int f(y)\left(b(y) n \mathrm{~d} v_{n-1}(y)+(D+d(y)) n \mathrm{~d} v_{n+1}(y)\right) \\
& \quad+\int f(y)(\lambda-(b(y)+D+d(y)) n) \mathrm{d} v_{n}(y)
\end{aligned}
$$

It is not difficult to construct a sequence of functions $\left(f_{q}: q \in \mathbb{N}^{*}\right)$ contained in $C^{\infty}$ with support in a fixed, small enough neighborhood of $y_{j}$ and such that

$$
\begin{gathered}
f_{q}^{\prime}\left(y_{j}\right)=1, \quad \lim _{q \rightarrow \infty} \sup _{y \in \mathbb{R}_{+}}\left|f_{q}(y)\right|=0, \quad \sup _{y \in \mathbb{R}_{+}}\left|f_{q}^{\prime}(y)\right| \leq 1 \quad \text { for all } q, \\
\\
\text { and } \lim _{q \rightarrow \infty}\left|f_{q}^{\prime}(y)\right|=0 \quad \text { for all } y \neq y_{j} .
\end{gathered}
$$

This leads to a contradiction, when we take $f=f_{q}$ in (5.3) and make $q$ tend to $\infty$.

Now, again using (5.2), we easily deduce the boundedness of the density of $v_{n}$ outside a neighborhood of $\left\{y_{n}, 0\right\}$.

Lemma 5.4. The probability measure $v_{n}$ cannot have a Dirac mass in $y_{n}$.

Proof. Assume that it does. Then in a neighborhood of $y_{n}$, by using (5.1), since $c_{j}^{n}=0$ for $j=0, n$, and by writing $c_{n}=c_{n}^{n}$, we can write

$$
\mathrm{d} v_{n}=c_{0}^{n} \delta_{0}+c_{n} \delta_{y_{n}}+u_{n}(y) \mathrm{d} y .
$$

Let $f$ be a function $C^{\infty}$ with compact support containing $y_{n}$ but that does not contain 0 . We have

$$
\begin{aligned}
-\int f^{\prime}(y)\left(D\left(y^{*}-y\right)-n \tilde{b}(y)\right) u_{n}(y) \mathrm{d} y \\
=\int f(y)\left(b(y) n \mathrm{~d} v_{n-1}(y)+(D+d(y)) n \mathrm{~d} v_{n+1}(y)\right) \\
\quad+\int f(y)(\lambda-(b(y)+D+d(y)) n) u_{n}(y) \mathrm{d} y \\
\quad+c_{n} f\left(y_{n}\right)\left(\lambda-\left(b\left(y_{n}\right)+D+d\left(y_{n}\right)\right) n\right) .
\end{aligned}
$$

We now construct a sequence $\left(f_{q}: q \in \mathbb{N}^{*}\right)$ of $C^{\infty}$-functions with support in a fixed, small enough neighborhood of $y_{n}$ such that, for some constant $C^{\prime}$,

$$
f_{q}\left(y_{n}\right)=1 \quad \text { for all } q, \quad \sup _{y \in \mathbb{R}_{+}}\left|f_{q}(y)\right|=1, \quad \lim _{q \rightarrow \infty}\left|f_{q}(y)\right|=0 \quad \text { for all } y \neq y_{n},
$$


and

$$
\sup _{y \in \mathbb{R}_{+}}\left|y-y_{n}\right|\left|f_{q}^{\prime}(y)\right| \leq C^{\prime} \quad \text { for all } q, \quad \lim _{q \rightarrow \infty}\left(y-y_{n}\right) f_{q}^{\prime}(y)=0 \quad \text { for all } y \neq y_{n} .
$$

Such a sequence can be easily constructed.

Recall that $v_{n-1}$ and $v_{n+1}$ are absolutely continuous with $C^{\infty}$-density near $y_{n}$. We conclude that, if $c_{n} \neq 0$,

$$
\lambda=\left(b\left(y_{n}\right)+D+d\left(y_{n}\right)\right) n .
$$

In the case where $d$ is constant, it is known that the eigenvalue $\lambda$ satisfies $\lambda=\kappa_{n}(D+d)<$ $D+d$, and so it is strictly less than $\left(b\left(y_{n}\right)+D+d\left(y_{n}\right)\right) n$ and we obtain a contradiction. If $d$ is not constant, the proof of the contradiction is more intricate.

Returning to (5.2) for $u_{n}$ in a neighborhood of $y_{n}$ but outside that point, we obtain

$$
\partial_{y}\left(G_{n}(y) u_{n}(y)\right)=f_{n}(y) G_{n}(y) u_{n}(y)+h_{n}(y),
$$

where (see Lemma 3.1)

$$
G_{n}(y)=D\left(y^{*}-y\right)-n \tilde{b}(y)=\beta_{n}\left(y-y_{n}\right)+\mathcal{O}\left(\left(y-y_{n}\right)^{2}\right)
$$

with $\beta_{n}<0$,

$$
f_{n}(y)=n \frac{b\left(y_{n}\right)-b(y)+d\left(y_{n}\right)-d(y)}{G_{n}(y)}=\mathcal{O}(1),
$$

and

$$
h_{n}(y)=b(y) n u_{n-1}(y)+(D+d(y)) n u_{n+1}(y) .
$$

Recall that $h_{n}(y)$ is $C^{\infty}$ near $y_{n}$. The only solution which is integrable near $y_{n}$ is given by

$$
u_{n}(y)=\frac{1}{G_{n}(y)} \exp \left(\int_{y_{n}}^{y} f_{n}(s) \mathrm{d} s\right) \int_{y_{n}}^{y} \exp \left(-\int_{y_{n}}^{s} f_{n}(w) \mathrm{d} w\right) h_{n}(s) \mathrm{d} s .
$$

If $h_{n}>0$ on a subset of positive measure of a small neighborhood of $y_{n}$, we have $u_{n}<0$, which is a contradiction. Therefore, $h_{n}$ must vanish on both sides of $y_{n}$. By the above result on the support, we conclude that $u_{n}$ vanishes in a neighborhood of $y_{n}$ as well as all the $v_{j}$ with $j \neq n$ (see Lemma 5.2). In particular, if we consider the equation for $v_{n+1}$ in this neighborhood (see (5.2)), we obtain

$$
0=b\left(y_{n}\right) c_{n} \delta_{y_{n}}
$$

which contradicts $c_{n} \neq 0$.

Theorem 5.2. On $\left(0, y_{1}\right)$, the density of $v_{n}$ satisfies $u_{n}>0$ except perhaps in $y_{n}$.

The proof uses two lemmas.

Lemma 5.5. If $v_{n+1}$ or $v_{n-1}$ has a support dense in $\left(0, y_{1}\right)$ then $u_{n}$ can be 0 only in $y_{n}$.

Proof. The function $G_{n}$ has a simple 0 in $y_{n}$. Assume that, for $z \in\left(0, y_{1}\right), z \neq y_{1}$, $u_{n}(z)=0$ (and $G_{n}(z) \neq 0$ ).

Computation as in the proof of Lemma 5.4 gives

$$
u_{n}(y)=\frac{1}{G_{n}(y)} \exp \left(\int_{z}^{y} f_{n}(s) \mathrm{d} s\right) \int_{z}^{y} \exp \left(-\int_{z}^{s} f_{n}(w) \mathrm{d} w\right) h_{n}(s) \mathrm{d} s .
$$

The conclusion follows. 
Let us show that the process is irreducible up to extinction and before $y_{1}$.

Lemma 5.6. Starting from any initial condition on $\mathbb{N}^{*} \times\left(0, y_{1}\right)$ the process $Z$ has dense support on $\mathbb{N} \times\left(0, y_{1}\right)$, that is, for all $m \in \mathbb{N}$, all $y^{\prime} \in\left(0, y_{1}\right)$, and all $\gamma>0$, there exists $t\left(y^{\prime}\right)>0$ such that, for all $t>t\left(y^{\prime}\right)$ and all $\left(y_{0}, n_{0}\right) \in \mathbb{N}^{*} \times\left(0, y_{1}\right)$,

$$
\mathbb{P}_{\left(n_{0}, y_{0}\right)}\left(N(t)=m, Y(t) \in\left(y^{\prime}-\gamma, y^{\prime}+\gamma\right)\right)>0 .
$$

Proof. In the proof we will assume that $y_{0}<y^{\prime}$ (the case $y_{0} \in\left(y^{\prime}, y_{1}\right)$ is shown similarly). Let $\beta>0$ be smaller than $\min \left(y_{0}, y^{\prime}-y_{0}\right) / 2$, and let $\tilde{t}>0$ be fixed. Then there exists $\varepsilon=\varepsilon\left(n_{0}, \beta\right)$ such that the following event has a strictly positive probability.

- On the interval time $[0, \varepsilon]$ there are exactly $n_{0}-1$ deaths and there is no other jump of $N$, and so $N(t)$ decreases from $n_{0}$ to $N(\varepsilon)=1$.

- $Y(\varepsilon)$ belongs to $(Y(0)-\beta, Y(0)+\beta)$.

- On the interval of time $[\varepsilon, \varepsilon+\tilde{t}]$ there is no jump of $N$ (no birth and no death).

- On the interval of time $[\varepsilon+\tilde{t}, \varepsilon+\tilde{t}+\beta]$ there are exactly $m-1$ births and no other jump when $m>1$, there is no jump if $m=1$, or there is a unique death and no other jump when $m=0$.

- $\mid Y(\varepsilon+\tilde{t})+\beta)-Y(\varepsilon+\tilde{t}) \mid<\gamma / 2$.

For $t \in[\varepsilon, \varepsilon+\tilde{t})$, we have $N(t)=1$. Then in this interval of time and before the process $Y(t)$ has attained $y^{\prime}$, the derivate

$$
\frac{\mathrm{d} Y(t)}{\mathrm{d} t}=D\left(y^{*}-Y(t)\right)-\tilde{b}(Y(t))
$$

is bounded below by $D\left(y^{*}-y^{\prime}\right)-\tilde{b}\left(y^{\prime}\right)$. Take $\tilde{t}=\left(y^{\prime}-Y(0)+\beta\right) /\left(D\left(y^{*}-y^{\prime}\right)-\tilde{b}\left(y^{\prime}\right)\right)$, and let us show that the number $t\left(y^{\prime}\right)=\varepsilon+\tilde{t}^{\prime}$ satisfies the property stated in the lemma. In fact, we have ensured that in a time smaller than or equal to $t\left(y^{\prime}\right)$ we have attained $\{m\} \times\left[y^{\prime}-\gamma, y^{\prime}+\gamma\right]$. For any time larger than $t\left(y^{\prime}\right)$, it suffices to modify slightly the above argument and allow a sequence of jumps up to the moment that $Y(t)$ has negative derivate and in this way we can postpone the time of attaining the set $\{m\} \times\left[y^{\prime}-\gamma, y^{\prime}+\gamma\right]$ from $t\left(y^{\prime}\right)$ to a prescribed time $t>t\left(y^{\prime}\right)$.

Proposition 5.2. For all $n \in \mathbb{N}^{*}$, the probability measure $v_{n}$ has a support dense in $\left(0, y_{1}\right)$.

Proof. Consider $\delta_{n} \otimes v_{n}$ the probability measure defined on $\mathbb{N} \times \mathbb{R}_{+}$given by $\delta_{n} \otimes v_{n}(\{m\} \times$ $B)=\delta_{n}(m) v_{n}(B)$ for all $B \in \mathscr{B}\left(\mathbb{R}_{+}\right)$. From Lemma 5.6 and the quasistationary distribution property (4.2), we have

$$
\begin{aligned}
\mathbb{P}_{\delta_{n} \otimes v_{n}}\left(N(0)=m, Y(0) \in\left(y^{\prime}-\gamma, y^{\prime}+\gamma\right)\right) \\
=\mathrm{e}^{-\lambda t} \mathbb{P}_{\delta_{n} \otimes v_{n}}\left(N(t)=m, Y(t) \in\left(y^{\prime}-\gamma, y^{\prime}+\gamma\right)\right) \\
=\mathrm{e}^{-\lambda t} \int \mathbb{P}_{\left(n, y_{0}\right)}\left(N(t)=m, Y(t) \in\left(y^{\prime}-\gamma, y^{\prime}+\gamma\right)\right) \mathrm{d} v_{n}\left(y_{0}\right) \\
>0 .
\end{aligned}
$$

Then the result follows. 


\section{Bound on the asymptotic survival rate}

Theorem 6.1. Let $\lambda$ be the exponential extinction rate associated with a quasistationary distribution $v$. Then

$$
\lambda<\inf _{n}\left(n\left(b\left(y_{n}\right)+D+d\left(y_{n}\right)\right)\right) .
$$

Proof. In Section 3 of [2], it was shown that this extinction rate is an eigenvalue of the dual problem associated with the probability measure

$$
\mathcal{L}^{\dagger} v=-\lambda v .
$$

For simplicity, we will prove inequality (6.1) in the case $n=1$. The general case proved in a similar way is left to the reader.

We introduce the notation

$$
G(y)=D\left(y^{*}-y\right)-\tilde{b}(y), \quad H(y)=D+d(y) .
$$

The quasistationary distribution equation for $n=1$ is given by

$$
-\frac{\mathrm{d}}{\mathrm{d} y}\left(G(y) u_{1}(y)\right)-(b(y)+H(y)) u_{1}(y)+2 H(y) u_{2}(y)+\lambda u_{1}(y)=0 .
$$

Let $a \in\left(0, y_{1}\right)$. We have, for $y \in\left(0, y_{1}\right)$,

$$
\begin{aligned}
& \frac{\mathrm{d}}{\mathrm{d} y}\left(G(y) u_{1}(y) \exp \left(\int_{a}^{y} \frac{b(s)+H(s)-\lambda}{G_{1}(s)} \mathrm{d} s\right)\right) \\
& =\exp \left(\int_{a}^{y} \frac{b(s)+H(s)-\lambda}{G(s)} \mathrm{d} s\right) 2 H(y) u_{2}(y)
\end{aligned}
$$

and integrating between $a$ and $y$ yields

$$
\begin{aligned}
G(y) & u_{1}(y) \exp \left(\int_{a}^{y} \frac{b(s)+H(s)-\lambda}{G(s)} \mathrm{d} s\right)-G(a) u_{1}(a) \\
& =2 \int_{a}^{y} \exp \left(\int_{a}^{\sigma} \frac{b(s)+H(s)-\lambda}{G(s)} \mathrm{d} s\right) H(\sigma) u_{2}(w) \mathrm{d} w \\
& \geq 0 .
\end{aligned}
$$

Therefore,

$$
u_{1}(y) \geq \frac{G(a) u_{1}(a)}{G(y)} \exp \left(-\int_{a}^{y} \frac{b(s)+H(s)-\lambda}{G(s)} \mathrm{d} s\right) .
$$

Let us study more carefully the quantity $\exp \left(-\int_{a}^{y}((b(s)+H(s)-\lambda) / G(s)) \mathrm{d} s\right)$. We recall that $G$ only vanishes at $y_{1}$ and that $G$ is decreasing since $b$ is increasing. By a simple computation we obtain

$$
\frac{b(s)+H(s)-\lambda}{G(s)}=\frac{b\left(y_{1}\right)+H\left(y_{1}\right)-\lambda}{\left(s-y_{1}\right) G^{\prime}\left(y_{1}\right)}+\mathcal{O}(1),
$$

and, thus,

$$
\int_{a}^{y} \frac{b(s)+H(s)-\lambda}{G(s)} \mathrm{d} s=\frac{b\left(y_{1}\right)+H\left(y_{1}\right)-\lambda}{G^{\prime}\left(y_{1}\right)} \log \left|y-y_{1}\right|+\mathcal{O}(1) .
$$


Finally, since $G\left(y_{1}\right)=0$ and $G^{\prime}\left(y_{1}\right)<0$, we obtain, for $y \leq y_{1}$,

$$
G(y)=\left(y-y_{1}\right) G^{\prime}\left(y_{1}\right)+\mathcal{O}\left(\left(y-y_{1}\right)^{2}\right)=\left|y-y_{1}\right|\left(\left|G^{\prime}\left(y_{1}\right)\right|+\mathcal{O}\left(\left|y-y_{1}\right|\right)\right) .
$$

Therefore,

$$
\begin{aligned}
u_{1}(y) & \geq \frac{G(a) u_{1}(a)}{G(y)}\left|y-y_{1}\right|^{-\left(b\left(y_{1}\right)+H\left(y_{1}\right)-\lambda\right) / G^{\prime}\left(y_{1}\right)} \mathrm{e}^{\mathcal{O}(1)} \\
& \geq \frac{G(a) u_{1}(a)}{\left|y-y_{1}\right|\left|G^{\prime}\left(y_{1}\right)\right|}\left|y-y_{1}\right|^{-\left(b\left(y_{1}\right)+H\left(y_{1}\right)-\lambda\right) / G^{\prime}\left(y_{1}\right)} \mathrm{e}^{\mathcal{O}(1)} .
\end{aligned}
$$

As we have $u_{1}(a)>0$ by Theorem 5.2 , the integrability of $u_{1}$ on $\left[0, y_{1}\right]$ implies that

$$
1+\frac{b\left(y_{1}\right)+H\left(y_{1}\right)-\lambda}{G^{\prime}\left(y_{1}\right)}<1 .
$$

We therefore finally obtain

$$
\lambda<b\left(y_{1}\right)+H\left(y_{1}\right)
$$

\section{Acknowledgements}

The authors are grateful to the anonymous referee for his/her comments and references that greatly helped to improve the presentation of this article. The authors acknowledge the support of CMM-BASAL CONICYT, MathAmsud, and ECOS projects. They are also grateful to CMAP and CPHT, École Polytechnique, for their hospitality.

\section{References}

[1] Campillo, F., Joannides, M. and Larramendy-Valverde, I. (2011). Stochastic modeling of the chemostat. Ecological Modelling 15, 2676-2689.

[2] Collet, P., Méléard, S., Martínez, S. and San Martín, J. (2011). Quasi-stationary distributions for structured birth and death processes with mutations. Prob. Theory Relat. Fields 151, 191-231.

[3] Crump, K. S. ANd O'Young, W.-S. C. (1979). Some stochastic features of bacterial constant growth apparatus. Bull. Math. Biol. 41, 53-66.

[4] De Leenheer, P., Li, B. And Smith, H. L. (2003). Competition in the chemostat: some remarks. Canad. Appl. Math. Quart. 11, 229-248.

[5] Dykhuizen, D. E. And Hartl, D. L. (1983). Selection in chemostats. Microbiol. Rev. 47, 150-168.

[6] Flegr, J. (1997). Two distinct types of natural selection in turbidostat-like and chemostat-like ecosystems. J. Theoret. Biol. 188, 121-126.

[7] Masci, P., Bernard, O. and Grognard, F. (2008). Continuous selection of the fastest growing species in the chemostat. In 17th IFAC World Congress, pp. 9707-9712.

[8] Masci, P., Grognard, F., Benoît, E. and Bernard, O. (2012). Competition between phytoplankton and bacteria: exclusion and coexistence. Res. Rep. RR-8038, INRIA.

[9] Monod, J. (1950). La technique de culture continue. Ann. Inst. Pasteur 79, 390 -410.

[10] Novick, A. ANd SzIlard, L. (1950). Description of the chemostat. Science 112, 715-716.

[11] Novick, A. AND SzILARD, L. (1950). Experiments with the chemostat on spontaneous mutations of bacteria. Proc. Nat. Acad. Sci. USA 36, 708-719.

[12] Smith, H. and Waltman, P. (1995). The Theory of the Chemostat. Cambridge University Press.

[13] VAN DooRn, E. A. (1991). Quasi-stationary distributions and convergence to quasi-stationarity of birth-death processes. Adv. Appl. Prob. 23, 683-700. 